\title{
USE OF LIDAR DERIVED OPTICAL EXTINCTION AND BACKSCATTERING COEFFICIENTS NEAR CLOUD BASE TO EXPLORE AEROSOL-CLOUD INTERACTIONS
}

\author{
Zaw Han ${ }^{1 *}$, Yonhgua Wu ${ }^{1}$, Barry Gross ${ }^{1}$, Fred Moshary ${ }^{1}$ \\ ${ }^{1}$ Optical Remote Sensing Lab, City College of New York, USA, *Email: zhan@ccny.cuny.edu
}

\begin{abstract}
Combination of microwave radiometer (MWR) and mutlifilter rotating shadowband radiometer (MFRSR) measurement data together with SBDART radiative transfer model to compute cloud optical depth (COD) and cloud droplet effective radius $\left(\mathrm{R}_{\mathrm{eff}}\right)$. Quantify the first aerosol indirect effect using calculated $\mathrm{R}_{\text {eff }}$ and aerosol extinction from Raman lidar measurement in urban coastal region. Illustrate comparison between ground-based and satellite retrievals. Demonstrate relationship between surface aerosol $\left(\mathrm{PM}_{2.5}\right)$ loading and $\mathrm{R}_{\text {eff. }}$ We also explain the sensitivity of aerosol-cloud-index (ACI) depend on the aerosol layer from cloud base height. Potential used of less noisy elastic backscattering to calculate the ACI instead of using Raman extinction. We also present comparison of elastic backscattering and Raman extinction correlation to $\mathrm{R}_{\text {eff. }}$
\end{abstract}

\section{INTRODUCTION}

It is generally understood that recent changes in climate have impacted the Earth's biosphere. Greenhouse gases play a significant role in the overall global energy balance, but other effect such as aerosols and the interaction of aerosols with clouds are much less understood. These uncertainties are the main source of uncertainty in the overall energy balance. In particular, the aerosol direct effect that is defined as the solar radiation, which is either absorbed or scattered by aerosol particles is estimated to partially compensate for the global warming due to greenhouse gas forcing but the magnitude of this effect is difficult to estimate due to the variety of aerosol properties that can either act as a cooling mechanism or a warming mechanism.

Far more complex are the aerosol-cloud interactions in which aerosols are thought to modify the microphysical and therefore the radiative properties and lifetime of clouds.
Generally, the aerosol can affect on cloud directly and indirectly, and consequences of the effects that lead to alter the climate condition in the local, regional, and global scale. Feingold quantified the aerosol indirect effect using models and radar reflectivity for cloud mask, Raman LIDAR for aerosol extinction, microwave radiometer for integrated liquid water path, combination of radar radiometer for cloud droplet effective radius at ARM CART site [1]. Additionally, Min propose the new technique for simultaneously retrieving cloud optic properties $\left(\tau_{\text {cod }}, R_{\text {eff }}\right)$ and LWP using radiative transfer model simulation [2]. In this paper, we provide the first results of aerosol-cloud interaction in an urban coastal environment (NYC).

\section{METHODOLOGY}

In order to measure the cloud and aerosol properties simultaneously, we make use of the technique discussed in [3]. In particular, a combination of a microwave radiometer (MWR) and the multi filter rotating shadow band radiometer (MFRSR) are needed for retrievals of cloud optical depth and cloud droplet effective radius. Basically, we used SBDART model to create look-up-table (LUT), HYSPLIT model to determine origin of air particles, and ground based instruments retrieval to calculate aerosol extinction coefficients and cloud optic properties. SBDART is a FORTRAN source code run on LINUX/UNIX that computes plane-parallel radiative transfer in clear and cloudy sky condition within the Earth's atmosphere and at the surface.

In this study, atmospheric transmittance is the quantity that can be measured and depends critically on cloud optical properties $\left(\tau_{\text {cod }}, R_{\text {eff }}\right)$ as well as the observed solar zenith angle. Figure $1 \& 2$ illustrate that the behavior of cloud optical depth and inverse transmittance with varying cloud droplet effective radius with fixed solar zenith angle and varying solar zenith angle with fixed cloud droplet effective radius. 


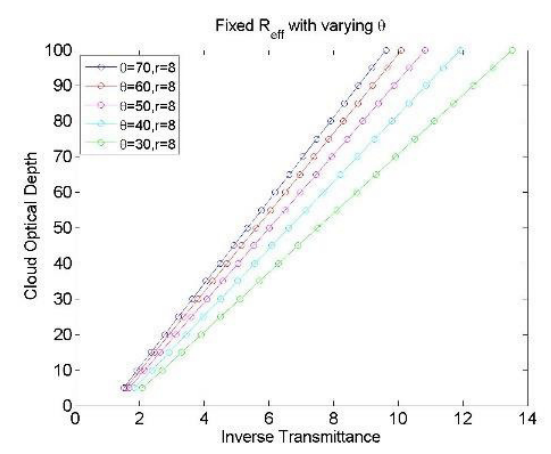

Figure 1. Fixed $\mathrm{R}_{\mathrm{eff}}$ with varying solar zenith angle.

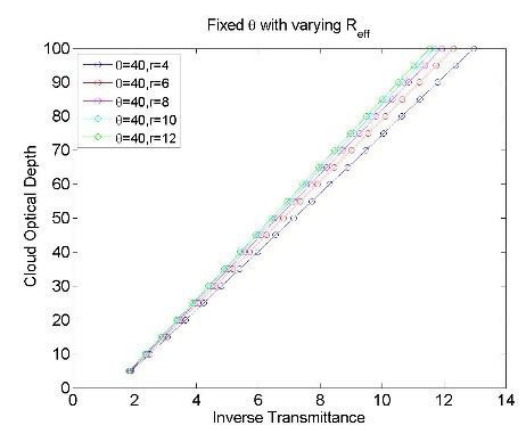

Figure 2. Fixed solar zenith angle with varying $R_{\text {eff. }}$.

The retrieval of cloud properties requires the simultaneous retrieval of cloud diffuse transmittance and liquid water path. The cloud diffuse transmittance is obtained by eq. 1 using the $415 \mathrm{~nm}$ channel of MFRSR. In order to use MFRSR, an estimation of the Top of Atmosphere (TOA) irradiance or calibration constant $\left(I_{0}\right)$ must be made. In particular, the atmospheric transmittance is evaluated using $415 \mathrm{~nm}$ channel because it is insensitive to absorption by ozone and atmospheric gases, suitable for natural surface, and low surface albedo.

$T_{\text {diff }}=\frac{I_{\text {diff }}}{I_{0} \cos (\theta)}$

In order to calculate the cloud optical depth and cloud droplet effective radius, the other crucial parameter is integrated liquid water path that can be obtained from MWR. In preliminary calculations, we made using of the default setting from the Radiometric Corporation on their Neural Network (NN) method.

Concisely, microwave radiometer (MP3000A) used four points nonlinear model for brightness temperature and surface meteorological data which is defined as level 1 product. Then train by neural network to retrieve level 2 product. Level
2 product consist of profiling and column integrated liquid water path.

Inauspiciously, since no other independent ground based measurements exist for integrated liquid water path validation, we decided to compare against a significantly different dual channel method [4] to retrieve integrated liquid water path and water vapor so that we compare two retrievals method to assess the accuracy of liquid water path.

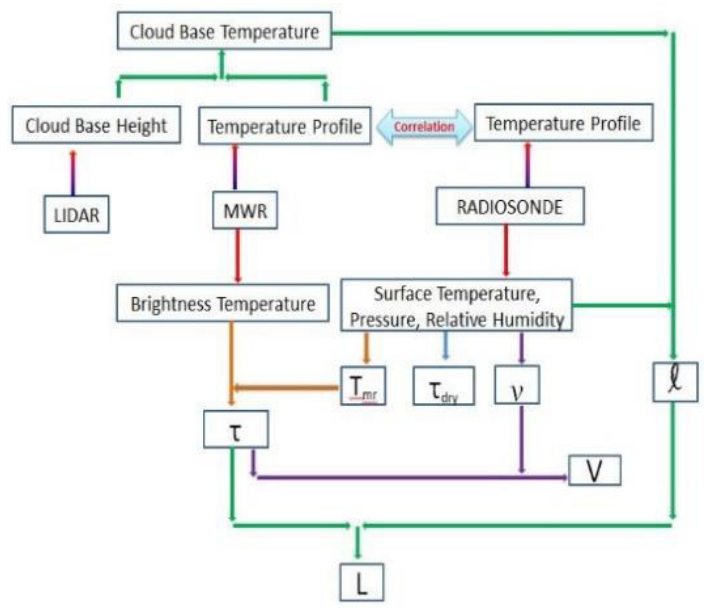

Figure 3. Dual-Channel LWP retrieval algorithm.

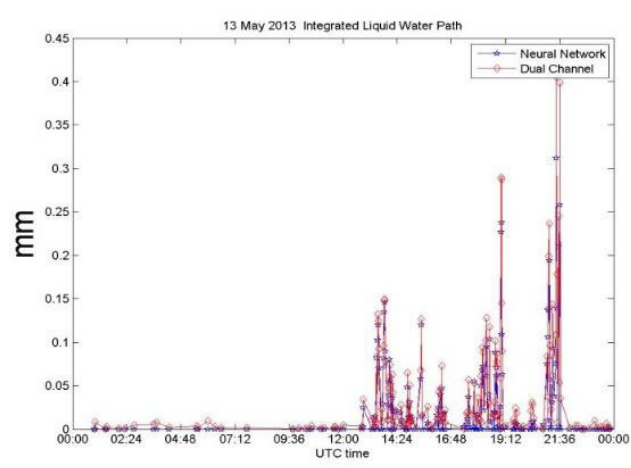

Figure 4. Integrated Liquid Water Path (mm) retrieved by dual channel and default neural network algorithms.

Once the cloud properties are obtained, we use Raman LIDAR to derive aerosol extinction coefficients $\left(\alpha_{\mathrm{ext}}\right)$ near cloud base which serve as a proxy to the aerosol number density. In particular, the following Raman LIDAR eq. (2) is used to compute $\alpha_{\text {ext }}$ below cloud.

$\alpha_{\text {ext }}\left(\lambda_{L}, z\right)=\frac{\frac{d}{d z}\left[\ln \frac{N(z)}{z^{2} P(z)}\right]-\alpha_{m o l}\left(\lambda_{L}, z\right)-\alpha_{m o l}\left(\lambda_{R}, z\right)}{1+\frac{\lambda_{L}}{\lambda_{R}}}$ 


\section{RESULTS}

This result is clearly consistent with the aerosol indirect effect. In this example, we also note that the DC method actually seems to outperform the $\mathrm{NN}$ approach. This can be attributed to the strict requirement of Microwave Brightness Channels which results in a smaller number of overall LWP retrievals and therefore less Cloud $\mathrm{R}_{\text {eff }}$ measurements. Even so, the overall consistency between the two approaches can be seen by the general good agreement with the ACI values.

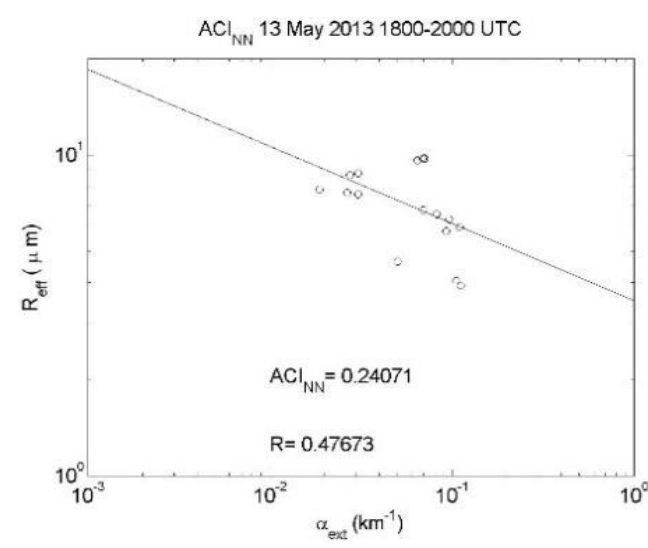

Figure 5. ACI using NN method.

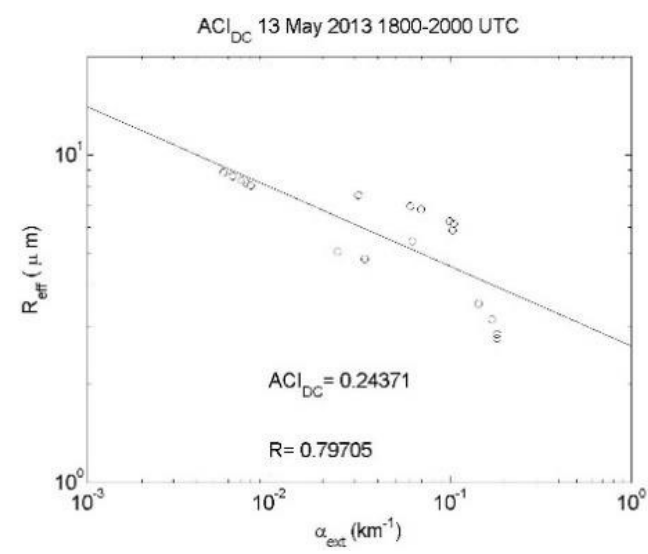

Figure 6. ACI using DC method.

In getting these results, care was taken to look at the underlying aerosol as close to the cloud base as possible without including any of the cloud fields. In this case, we see that at least 100-150 meters gap was necessary to guarantee that no cloud contamination was made in the aerosol estimates. The sensitivity of this measurement to the vertical layer chosen can be seen in figure $7 \& 8$ where both the ACI coefficient and correlation (for both DC and $\mathrm{NN}$ approaches) are plotted as a function of the aerosol reference height.

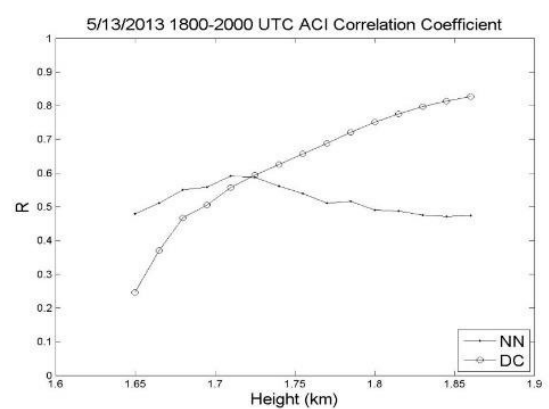

Figure 7. Variation of correlation coefficient (R) of $\mathrm{ACI}$ as a function of the aerosol loading (height).

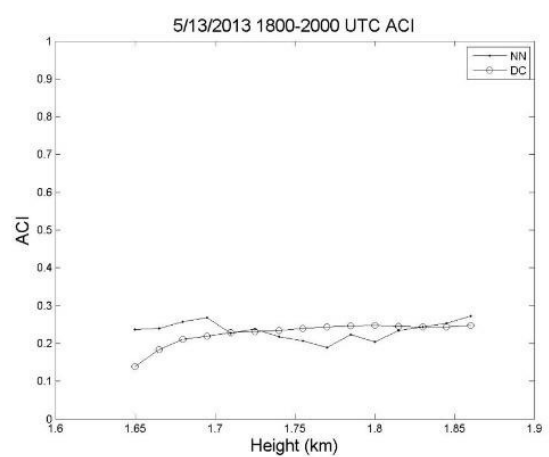

Figure 8. ACI within the theoretical limit as a function of height (aerosol range).

For using elastic backscattering at 1064 $\mathrm{nm}$, we first explore the relationship between lidar derived aerosol extinction and number concentration from AERONET. Figure $9 \& 10$ show that behavior between number concentration against extinction and backscattering. To see the results using backscatter at $1064 \mathrm{~nm}$, we reexamine the two cases for both NN and DC cloud droplet effective radius retrievals using extinction and backscattering. Table 1 represent correlation coefficient comparison of extinction $(\alpha)$ and backscattering $(\beta)$ using neural network and dual channel retrieval algorithm.

\begin{tabular}{|l|l|l|l|l|}
\hline \multirow{2}{*}{$\begin{array}{l}\text { Retrieval } \\
\text { method }\end{array}$} & \multicolumn{2}{|l|}{ Morning } & \multicolumn{2}{l|}{ Evening } \\
\cline { 2 - 5 } & $\alpha$ & $\beta$ & $\alpha$ & $\beta$ \\
\hline NN & 0.83 & 0.99 & 0.47 & 0.62 \\
\hline DC & 0.79 & 0.79 & 0.91 & 0.98 \\
\hline
\end{tabular}




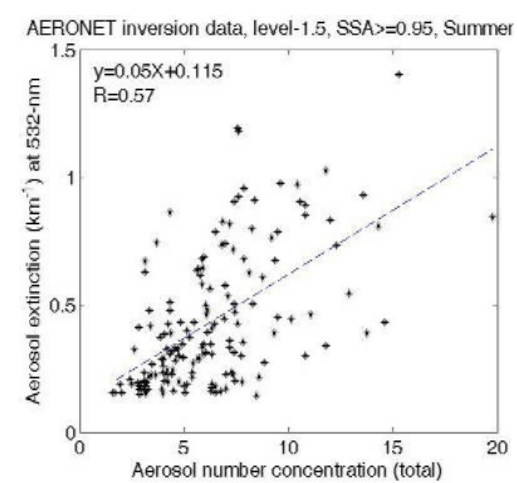

Figure 9. Aerosol extinction and number concentration.

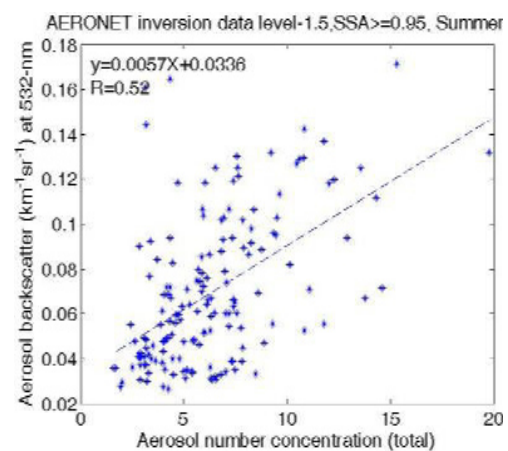

Figure 10. Aerosol backscattering and number concentration.

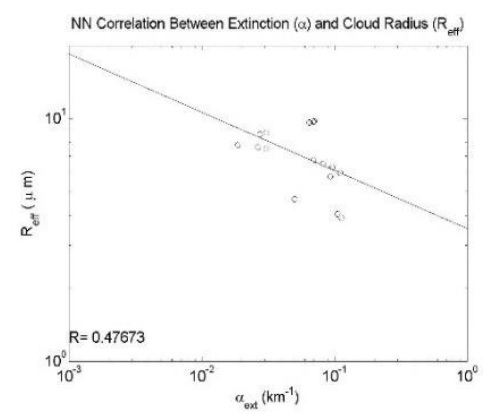

Figure 11. NN retrieval (1800-2000 case) of cloud droplet effective radius changed by aerosol extinction coefficient

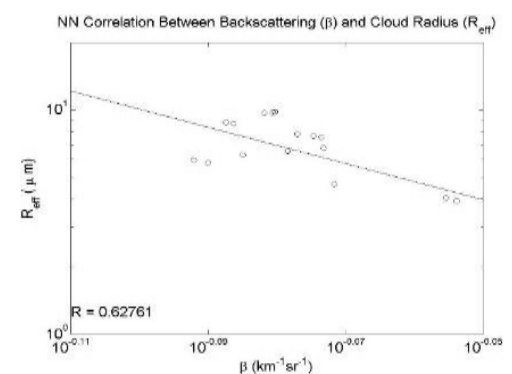

Figure 12. NN retrieval (1800-2000 case) of cloud droplet effective radius modified by aerosol.

\section{CONCLUSIONS}

In this paper, we have made a preliminary investigation of the potential of quantifying and generally observing Aerosol Cloud Interactions. In particular, the LWP retrieval using DC agrees well with the $\mathrm{NN}$ when both data are available, but allows for more retrievals which improves the overall statistics when using allowing for a larger number of data retrievals. We also demonstrated that the observation of the ACI effect is very sensitive to the distance from the cloud base. We found the ACI correlation will persist for as much as 200 meters below the cloud.

Finally, we explored the potential of using the backscattering coefficient at $1064 \mathrm{~nm}$ as a proxy for fine mode particulates. Then, for all the cases where the extinction was shown to be a useful indicator of ACI, the backscatter approach was used and we found that the backscatter signal has a generally better correlation.

\section{ACKNOWLEDGEMENT}

This work is partially supported by the NOAA CREST Center under contract NOAA \#NA17AE1625.

\section{REFERENCES}

[1] Feingold G., Eberhard W.L., Veron D.E., Previdi M., "First measurements of the Twomey indirect effect using ground-based remote sensors", Geophys. Res. Lett., (2003).

[2] Min Q., and M. Duan, "Simultaneously retrieving cloud optical depth and effective radius for optically thin clouds", J. Geophys. Res., 110, D21201 (2005).

[3] Madhavan B.L., He Y., Wu Y., Gross B., Moshary F., and Ahmed S., "Development of a ground based remote sensing approach for direct evaluation of aerosol-cloud interaction", Atmosphere, 3, 468-494 (2012).

[4] Liljegren J.C., E.E. Clothiaux, G.G. Mace, S. Kato, and X. Dong, "A new retrieval for cloud liquid water path using a ground-based microwave radiometer and measurements of cloud temperature", J. Geophys. Res., 10 (D13), 1448514500, July 16 (2001). 